\title{
What to expect from HER-2 directed therapies in advanced gastric cancer?
}

Faysal DANE

\begin{abstract}
Gastric cancer is the second most common cause of cancer related death worldwide. Over $20 \%$ of the advanced gastric cancer are considered to be HER-2 positive. Studies investigating the prognosis of HER-2 positive advanced gastric cancer revealed conflicting results. Trastuzumab, a monoclonal antibody against HER-2, has shown a significant clinical activity in HER-2 positive gastric cancer patients. In this review, I will briefly summarize the clinical studies of anti-HER-2 therapies performed in HER-2 positive gastric carcinoma.
\end{abstract}

Keywords: Gastric cancer, HER-2/neu, Targeted therapy

\section{Introduction}

Mortality rate of upper gastrointestinal (GI) tract malignancies is very high. Survival rates for gastric cancers are among the worst reported for any malignancy. The reason for poor prognosis is considered to be due to early metastatic dissemination tendency of upper GI cancers $[1,2]$. Although gastric cancer incidence is much lower in western Europe and United States than in eatern Asia, gastroesophageal, cardia and lower esophageal cancer incidence has incerased in Europe and in United States.

Faysal Dane, MD., (西)

Division of Medical Oncology

Department of Internal Medicine

Marmara University School of Medicine

Mimar Sinan Caddesi No: 41 Üst Kaynarca Fevziçakmak Mah. / Pendik / Istanbul, Turkey

e-mail:faysaldane@yahoo.com
In general, curative approach is not possible for locally advanced unresectable and metastatic gastroesophageal cancers. Thus, symptom palliation and survival prolongation are the main goals of treatment in these advanced cancers. Both local and/or systemic palliative modalities for advanced gastro-esophageal cancers may be applied for symptoms relief. Endoscopic, surgical, or radiotherapeutic modalities can successfully be applied for symptoms such as pain, obstruction, perforation, or bleeding. Systemic therapy is the most effective treatment modality for patients with metastatic disease and it may adequately palliate cancer related symptoms and improve survival rates. Although, there are many randomized clinical trials, there is no consensus indicating the best regimen for the first line chemotherapy in advanced gastric cancer. Combination chemotherapy regimens are more effective than monotherapy in terms of response rates but only moderate improvements in overall survival have been reported. Despite many improvements in this cancer, even in patients treated with three drug combination chemotherapies, the median overall survival is less than a year in advanced gastric cancer [3,4]. As in other cancers, the addition of targeted drugs to the combination chemotherapies in advanced gastric cancer was hoped to improve survival rates in this poor prognosis cancer. Here in this overview, I will review the overexpression and or amplification of human epidermal growth factor receptor 2 (HER-2), and the efficacy of anti-HER-2 therapy in advanced gastric cancer.

\section{The human epidermal growth factor receptor-2 (HER-2)}

The HER-2 gene is located on chromosome 17q21. It is also known as HER-2/neu or ERBB-2. HER-2 is a 185 $\mathrm{Kd}$ glycoprotein having tyrosine kinase activity and is 
the product of the HER-2/neu oncogene [5]. It has been demonstrated that HER-2 is important in cell proliferation, differentiation, adhesion, invasion and metastasis, tumor induced neoangiogenesis, and survival via activation of the cellular pathways RAS-RAF-MEK-MAPK and PI3K/Akt/ mTOR.

\section{The rationale for anti-HER-2 therapy in gastric cancer}

Amplification of the c-erb-B-2 gene in a gastric cancer cell line MKN-7 was first described in 1986 by Yamamoto et al [6]. Overall, approximately 7 to 22 percent of esophagogastric cancers overexpress HER-2 [7-9]. HER2 overexpression was positive immunohistocemically in $17.6 \%$ of 3264 specimen. Concordance between FISH and IHC was $93.5 \%$ in 168 evaluable samples. Eleven samples were scored as FISH+ but IHC- or equivocal [10]. In the same study, HER-2 amplification was positive in 19.2\% of 1232 gastric cancer samples. HER-2 positivity is more common with intestinal-type than with diffuse-type gastric cancers or mixed (32\% vs. $6 \%$ vs. $20 \%$ p:0.001) [11]. Her- 2 overexpression is more common in esofagogastric junction than gastric tumors. Since HER-2 positive clones comprise only a small proportion of the tumor in gastric cancers, a high degree of intratumoral HER-2 heterogeneity has been reported in gastric cancer [10,12]. Tissue samples, specifically biopsies, may miss HER-2 overexpressed region of the tumor and therefore be interpreated as false negative due to this intratumoral heterogeneity.

The association between HER-2 overexpression or amplification and prognosis in esophagogastric cancer remains unknown. Retrospective evaluations of HER-2 expression and gene amplification in relation to prognosis for gastric/esophagogastric junction (EGJ) adenocarcinomas have been performed in at least seven studies of prospectively enrolled clinical trial cohorts [13-19]. The results are conflicting. Four studies found that HER-2 was not associated with prognosis. While two studies reported a significant positive association and another study showed a trend toward improved survival with HER-2 overexpression. In one study, HER-2 overexpression was associated with shorter survival, but only among patients who received adjuvant postoperative chemoradiotherapy after potentially curative resection. Additionally, in a metaanalysis containing 11337 patients from 49 gastric cancer studies (stage I to IV), patients with HER-2 overexpression had shorter five-year overall survival than HER-2 negative patients ( $42 \%$ vs. $52 \%)$ [20].

\section{Clinical data of anti-HER-2 therapy in advanced gastric cancer}

Trastuzumab - Today, all patients with advanced gastric cancer should be recommended for HER-2 testing. The benefit of trastuzumab in advanced HER-2 positive adenocarcinoma of the stomach or EGJ was addressed in the phase III ToGA trial (21). In this study, patients were randomised for standart chemotherapy (six courses of cisplatin plus either infusional 5-FU or capecitabine) with or without trastuzumab $(8 \mathrm{mg} / \mathrm{kg}$ loading dose, then $6 \mathrm{mg} / \mathrm{kg}$ every three weeks until disease progression). All tumors were screened for HER-2 status by both immunohistochemistry (IHC) and FISH. Patients were eligible if their tumor was positive by either IHC (defined as showing $3+$ expression) or FISH (defined as showing a HER2/CEP17 ratio of 2 or greater). All enrolled 594 patients were FISH positive, whereas protein expression by IHC varied (47 percent $3+$, 30 percent $2+$, and 22 percent 0 or $1+$ ). A $22.1 \%$ HER-2 positivity rate was reported, with the highest proportion in esophagogastric junction tumours $(33.2 \%$ compared to $20.9 \%$ in gastric tumours, $\mathrm{p}<0.001)$ and intestinal-type cancers $(32.2 \%$ compared to $6.1 \%$ of diffuse and $20.4 \%$ of mixed types, $\mathrm{p}<0.001)$. The study met the primary endpoint of improved overall survival, as well as secondary endpoints of improved progression-free survival and response rate. The objective response rate was significantly higher with trastuzumab ( $47 \%$ vs. $35 \%)$. At a median follow-up about 18 months, median overall survival was significantly better with trastuzumab (13.8 vs. 11.1 months). The toxicities in two arms were comparable, except that a higher number of trastuzumab-treated patients had grade 3 or 4 diarrhea ( $9 \%$ vs. $4 \%$ ) and an asymptomatic decrease in left ventricular ejection fraction (LVEF, $5 \%$ vs. $1 \%$ ). Only one patient developed grade 3 to 4 heart failure (versus two in the control group). Exploratory analysis in subgroups defined by protein expression suggested that trastuzumab was most effective in prolonging survival in the subgroup of patients with IHC $3+$ tumors (16 months vs. 11.8 months, hazard ratio (HR) for death $0.66,95 \%$ confidence interval (CI) 0.50-0.87), less effective in patients with IHC $2+$ tumors (HR 0.78, 95\% CI 0.55-1.10), and ineffective in those with HER-2 gene-amplified (ie, FISH-positive) but non-proteinexpressing (IHC 0 or $1+$ ) tumors.

Based upon these data, trastuzumab was approved in USA, in Europe, in Turkey, and in many other countries in combination with cisplatin and a fluoropyrimidine, for the treatment of patients with IHC3+ or IHC 2+/ FISH+ metastatic HER-2-overexpressing gastric or EGJ adenocarcinomas who have not received prior treatment for metastatic disease. 
Recently, in a phase 2 study, combination of trastuzumab and capecitabine-oxaliplatin was shown to be well tolerated and highly effective in patients with HER-2 positive advanced gastric cancer. In this study, investigators enrolled 55 HER2 positive previously untreated advanced gastric cancer patients. The primary end-point was the objective response rate, and secondary end-points included progression-free survival, overall survival and toxicity profiles. Confirmed objective response rate was $67 \%$ (95\% CI 54-80\%). After a median follow-up period of 13.8 months, median PFS and OS were 9.8 months and 21 months, respectively. Frequently encountered grade 3-4 toxicities included neutropenia (18\%), anaemia (11\%), and peripheral neuropathy (11\%). Although the study is a phase 2 study, over 20 months overall survival data implies that this regimen is very promising and thus may be investigated in further studies (22).

If a patient progresses while on a first-line trastuzumab based regimen for HER-2 positive gastric cancer, today, there is no data demonstrating the benefit of continuing trastuzumab with the second-line regimen.

Lapatinib - Lapatinib is an orally active small molecule inhibitor of both EGFR and HER-2. The activity of lapatinib has been studied in some phase II studies and modest single agent activity was observed. Most recently, the activiy of lapatinib in combination with chemotherapy in metastatic HER-2 positive gastric cancer has been evaluated in two phase III studies. In the double blind LOGIC trial, 545 patients were randomized (1:1) to capecitabine/oxaliplatin chemotherapy with or without lapatinib. In a preliminary report presented at 2013 ASCO annual meeting, the primary endpoint (overall survival of the patients who were centrally confirmed to be FISH positive for HER-2) was not met (HR for OS: 0.91, [95\% CI: 0.73; 1.12]; $\mathrm{p}=0.35$ ). Median survival was 12.2 versus 10.5 months in the lapatinib versus control arm, respectively. Subgroup analysis revealed that Asian patients (median survival 16.5 versus 10.9 months, HR 0.68 ) and those younger than 60 years (median survival 12.9 versus 9 months, HR 0.69) seemed to benefit from the addition of lapatinib (23). The addition of lapatinib increased toxicity such as diarrhea, rash, palmar-plantar erythrodysesthesias. TYTAN study compared adding lapatinib with paclitaxel versus paclitaxel alone in the second-line treatment of Asian patients. About 261 HER2-amplified patients were enrolled [24]. Median OS was 11.0 months for lapatinib/paclitaxel and 8.9 months for paclitaxel alone in the intent-to-treat population (HR: 0.84; $\mathrm{p}=0.2088$ ). In a preplanned subgroup analysis, median OS in HER-2 IHC 3+ subgroup was 14.0 months for lapatinib/ paclitaxel and 7.6 months for paclitaxel alone $(p=0.0176)$. Therefore, neither in patients with previously untreated advanced gastric cancer, nor in second line setting, benefit of adding lapatinib to chemotherapy (capecitabine/oxaliplatin and paclitaxel respectively) could be demonstrated. Because of the results of these two randomised phase III studies, until further information becomes available, lapatinib can not be recommended as initial therapy for the treatment of advanced esophagogastric cancer.

\section{Ongoing phase 3 studies targeting HER-2}

There are some phase II/III studies evaluating the clinical activity of pertuzumab or trastuzumab-emtansine (TDM1) in advanced HER-2 positive gastric cancer in the first and second line setting. In the JACOP study, an international double blind and placebo controlled phase III trial, patients with metastatic gastric or GEJ cancer are being randomized 1:1 to receive pertuzumab, trastuzumab and chemotherapy or the same regimen replacing pertuzumab with placebo [25]. The primary endpoint of the study is OS. GATSBY study is a phase II/III study comparing the safety and efficacy of TDM1 to taxanes in previously treated HER-2 positive gastric cancer [26]. In the phase II TOXAG study, safety and efficacy of trastuzumab plus capesitabine/oxaliplatin and capesitabine-radiation is evaluated in curatively resected HER-2 positive gastric cancer in the adjuvant setting [27].

\section{References}

1. Garcia M, Jemal A, Ward EM, et al. Global cancer facts and figures 2007. Atlanta, GA: American Cancer Society, 2007.

2. Kamangar F, Dores GM, Anderson WF. Patterns of cancer incidence, mortality, and prevalence across fi ve continents: defining priorities to reduce cancer disparities in different geographic regions of the world. J Clin Oncol 2006; 24: 2137-50. doi: 10.1200/ JCO.2005.05.2308

3. Cunningham D, Starling N, Rao S, et al. Capecitabine and oxaliplatin for advanced esophagogastric cancer. N Engl J Med 2008; 358:36. doi: 10.1056/NEJMoa073149.

4. Ajani JA, Moiseyenko VM, Tjulandin S, et al. Clinical benefit with docetaxel plus fluorouracil and cisplatin compared with cisplatin and fluorouracil in a phase III trial of advanced gastric or gastroesophageal cancer adenocarcinoma: the V-325 Study Group. J Clin Oncol 2007; 25:3205. doi: 10.1200/JCO.2006.10.4968

5. Schechter AL, Hung MC, Vaidyanathan L, et al. The neu gene: an erbB-homologous gene distinct from and unlinked to the gene encoding the EGF receptor. Science 1985; 229(4717):976-8. 
6. Yamamoto T, Ikawa S, Akiyama T, et al. Similarity of protein encoded by the human c-erb-B-2 gene to epidermal growth factor receptor. Nature 1986;319(6050):230-4.

7. Park YS, Hwang HS, Park HJ, et al. Comprehensive analysis of HER2 expression and gene amplification in gastric cancers using immunohistochemistry and in situ hybridization: which scoring system should we use? Hum Pathol 2012; 43:413. doi: 10.1016/j. humpath.2011.05.019.

8. Barros-Silva JD, Leitão D, Afonso L, et al. Association of ERBB2 gene status with histopathological parameters and disease-specific survival in gastric carcinoma patients. Br J Cancer 2009; 100:487. doi: 10.1038/ sj.bjc. 6604885 .

9. Baykara M, Benekli M, Ekinci O, Irkkan C,DemirciU, Karaca H, Unal O.U,Dane F, Akinci B, Turkoz F.P. Clinical significance of HER2 overexpression in gastric and gastro-esophageal junction cancers: Is there any discordance between IHC and FISH. ESMO 2012, abst:2778.

10. Hofmann M, Stoss O, Shi D, et al. Assessment of a HER2 scoring system for gastric cancer: results from a validation study. Histopathology 2008;52:797-805. doi:10.1111/j.1365-2559.2008.03028.x.

11. Bang Y, Chung H, Xu J, et al. Pathological features of advanced gastric cancer (GC): Relationship to human epidermal growth factor receptor 2 (HER2) positivity in the global screening programme of the ToGA trial (abstract). J Clin Oncol 2009; 27:215s.

12. Rüschoff J, Dietel M, Baretton G, et al. HER2 diagnostics in gastric cancer-guideline validation and development of standardized immunohistochemical testing. Virchows Arch 2010; 457:299. doi: 10.1007/ s00428-010-0952-2.

13. Gordon MA, Gundacker HM, Benedetti J, et al. Assessment of HER2 gene amplification in adenocarcinomas of the stomach or gastroesophageal junction in the INT-0116/SWOG9008 clinical trial. Ann Oncol 2013; 24:1754. doi: 10.1093/annonc/mdt106.

14. Terashima $M$, Kitada $K$, Ochiai $A$, et al. Impact of expression of human epidermal growth factor receptors EGFR and ERBB2 on survival in stage II/ III gastric cancer. Clin Cancer Res 2012; 18:5992.doi: 10.1158/1078-0432.CCR-12-1318.

15. Lordick F, Kang YK, Chung HC, et al. Capecitabine and cisplatin with or without cetuximab for patients with previously untreated advanced gastric cancer
(EXPAND): a randomised, open-label phase 3 trial. Lancet Oncol 2013; 14:490. doi: 10.1016/S14702045(13)70102-5.

16. Waddell T, Chau I, Cunningham D, et al. Epirubicin, oxaliplatin, and capecitabine with or without panitumumab for patients with previously untreated advanced oesophagogastric cancer (REAL3): a randomised, open-label phase 3 trial. Lancet Oncol 2013; 14:481. doi: 10.1016/S1470-2045(13)70096-2.

17. Van Cutsem E, de Haas S, Kang YK, et al. Bevacizumab in combination with chemotherapy as first-line therapy in advanced gastric cancer: a biomarker evaluation from the AVAGAST randomized phase III trial. J Clin Oncol 2012; 30:2119.doi: 10.1200/JCO.2011.39.9824.

18. Janjigian YY, Werner D, Pauligk C, et al. Prognosis of metastatic gastric and gastroesophageal junction cancer by HER2 status: a European and USA International collaborative analysis. Ann Oncol 2012; 23:2656. doi: 10.1093/annonc/mds 104

19. Okines AF, Thompson LC, Cunningham D, et al. Effect of HER2 on prognosis and benefit from perioperative chemotherapy in early oesophago-gastric adenocarcinoma in the MAGIC trial. Ann Oncol 2013; 24:1253. doi: 10.1093/annonc/mds622.

20. Chua TC, Merrett ND. Clinicopathologic factors associated with HER2-positive gastric cancer and its impact on survival outcomes--a systematic review. Int J Cancer 2012; 130:2845. doi: 10.1002/ijc.26292.

21. Bang YJ, Van Cutsem E, Feyereislova A, et al. Trastuzumab in combination with chemotherapy versus chemotherapy alone for treatment of HER2-positive advanced gastric or gastro-oesophageal junction cancer (ToGA): a phase 3, open-label, randomised controlled trial. Lancet 2010; 376:687. doi: 10.1016/S01406736(10)61121-X.

22. Ryu MH, Yoo C, Kim JG, Ryoo BY, Park YS, Park SR, Han HS, Chung IJ, Song EK, Lee KH, Kang SY, Kang YK. Multicenter phase II study of trastuzumab in combination with capecitabine and oxaliplatin for advanced gastric cancer. Eur J Cancer 2015;5:482-8. doi:10.1016/j.ejca.2014.12.015.

23. Hecht JR, Bang YJ, Qin S, et al. Lapatinib in combination with capecitabine plus oxaliplatin (CapeOx) in HER2positive advanced or metastatic gastric, esophageal, or gastroesophageal adenocarcinoma (AC): The TRIO013/LOGiC Trial. J Clin Oncol 31, 2013 (suppl; abstr LBA4001)

24. Satoh T, Xu RH, Chung HC, Sun GP, Doi T, Xu JM, 
Tsuji A, Omuro Y, Li J, Wang JW, Miwa H, Qin SK, Chung IJ, Yeh KH, Feng JF, Mukaiyama A, Kobayashi M, Ohtsu A, Bang YJ. Lapatinib plus paclitaxel versus paclitaxel alone in the second-line treatment of HER2amplified advanced gastric cancer in Asian populations: TyTAN--a randomized, phase III study. J Clin Oncol 2014;32:2039-49. doi: 10.1200/JCO.2013.53.6136.

25. A Study of Perjeta (Pertuzumab) in Combination With Herceptin (Trastuzumab) and Chemotherapy in Patients With HER2-Positive Metastatic Gastroesophageal Junction or Gastric Cancer. Available from: https://clinicaltrials.gov/ct2/show/
NCT01774786? term $=$ NCT01774786\&rank $=1$

26. A Study of Trastuzumab Emtansine versus Taxane in Patients with Advanced Gastric Cancer. Available from: https://clinicaltrials.gov/ct2/show/ NCT01641939?term $=$ NCT01641939\&rank $=1$

27. A Study of the Combination of Oxaliplatin, Capecitabine and Herceptin (Trastuzumab) and Chemoradiotherapy in The Adjuvant Setting in Operated Patients With HER2+ Gastric or GastroEsophageal Junction Cancer (TOXAG Study). Available from: https://clinicaltrials.gov/ct2/show/ NCT01748773?term $=$ NCT01748773\&rank $=1$ 